\title{
Analysis of Medical College Students' Sports Situation and Dependence on Mobile Phones
}

\author{
Junjie Yin \\ Luohe Medical College, Food Nutrition Department, Henan, China \\ $1079345161 @ q q . c o m$
}

Keywords: Mobile phone dependence; Sports exercise; Medical college students

\begin{abstract}
With portability and intelligence of mobile terminals, more and more Chinese dwellers own mobile phones. College students, active and easy to accept new things, get more information and convenience of social networking; also bear some negative impact on their physical and mental health. Firstly, find the current situation of the students' dependence on mobile phones and the corresponding adverse health effects. Secondly, discuss the relationship of mobile phone use habits and sports exercise habits. Finally, try to propose some strategies to change the negative influence.
\end{abstract}

\section{医学高专学生手机依赖与体育运动现状分析}

\author{
尹军杰 \\ 漯河医学高等专科学校食品营养系, 中国 河南 漯河 462002 \\ 1079345161@qq.com
}

摘要: 随着移动终端的便携化与智能化, 我国居民智能手机拥有量逐渐上升, 大学生是思想 活跃, 较能接受新鲜事物的群体, 手机给大学生带了获取信息, 社交的便利, 也给大学生身 心健康造成了一定影响。本文分析了医学高专学生手机依赖的情况, 了解学生对手机依赖的 现状, 对健康的不良影响, 探讨手机使用习惯与体育运动习惯存在的关系, 分析体育运动改 变这一现象负面作用的对策。

\section{关键词: 手机依赖; 体育运动; 医学高专; 学生}

\section{1. 引言}

智能手机智能化后因为有优秀的操作系统, 可自由安装各类软件, 并且完全大屏的全触屏式 操作这些特性, 成为人们获得网络信息及社会交流的主要设备。在尼尔森选取研究的 10 个国 家中, 智能手机普在中国及率分别为 $66 \%$, 只低于韩国 $67 \%$, 已经超越美国和英国的普及率。 大学生思维敏捷, 接受新生事物快, 追求时尚, 手机上网已经成为他们社交, 娱乐, 学习, 生活与工作的重要工具。但过度使用手机上网对他们身体健康, 心理健康, 以及现实生活中 的人际关系都产生了不良影响。与网络依赖相似, 现在大学生也出现了一定程度的手机依赖。 对手机的过于依赖体现了大学生内心的孤独, 社交圈子虚拟化, 不肯与其他人直接沟通, 或 者是恐惧交友，被人们称为 “低头族” [1-2]。

长时间使用手机, 辐射对人的视力产生伤害, 导致视力下降, 出现黄斑眼病; 另外, 长期依 赖手机, 会出现关节疼痛、手脚发麻、心悸、头晕、容易疲劳等病症, 严重危害人体的健康 [3]。高专学生群体有特殊的属性, 比着重点院校的学生在自控能力, 对学习的用功程度存在 差异, 但思想活跃, 热衷同龄交往、喜爱运动。医学专业对学生在教育标准上, 课程要求, 身心健康上有较高要求, 本文调查了医学专业高专学生使用手机的情况, 以了解学生使用手 机的情况, 手机使用对学生的身体健康影响, 学生运动习惯和手机依赖之间的规律, 以期为 通过运动增强学生体质, 改变手机使用的不良影响提供依据。 


\section{2. 调查对象与方法}

2. 1 . 调查

通过问卷调查, 于 2016 年 3 月对漯河医学高等专科学校护理 (37)、口腔 (83)、药学 (38)、食 品 (51) 四个系部在校生为调查对象。共发放问卷 209 份, 收回 209 份, 问卷有效回收率为 $100 \%$ 。 其中: 男生 36 人, 女生 173 人; 大一学生 191 人, 大二学生 18 人, 年龄范围在 $17-23$ 岁, 平均年龄 19.73 岁。调查表项目共有 13 项, 分别为性别、年龄、手机依赖度、认可的恢复方 式、上网意图、体育锻炼习惯评价、锻炼目的、锻炼效果等。

2.2. 数据分析

采用 SPSS 20.0 软件包进行统计分析, 采用均数、标准差、百分率等进行统计描述。

\section{3. 结果}

3.1. 学生手机使用情况

通过调查发现, 当代大学生使用手机情况普遍, 有高达 $65.55 \%$ 的学生认为手机已成为生活中 不可或缺的一部分，平均每天上网时间 $4.73 \mathrm{~h}$ 。在调查主观认为是否存在手机依赖时, $65.17 \%$ 的学生认可, $18.66 \%$ 的学生否认对手机存在依赖。调查学生使用手机时间时, 女生使用手机 的时间为平均 $4.9 \mathrm{~h}$, 显著多于男生的 $3.8 \mathrm{~h}$ 。在不同系部的学生之间, 使用手机的情况显著 不同, 其中护理系学生、药学系、食品营养系使用手机平均时间依次降低, 但之间差异不显 著，口腔系学生使用手机时间平均是 4 h，显著少于其它三个系部学生（Fig. 1)。

A

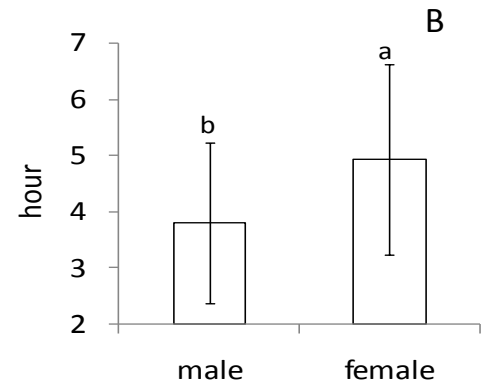

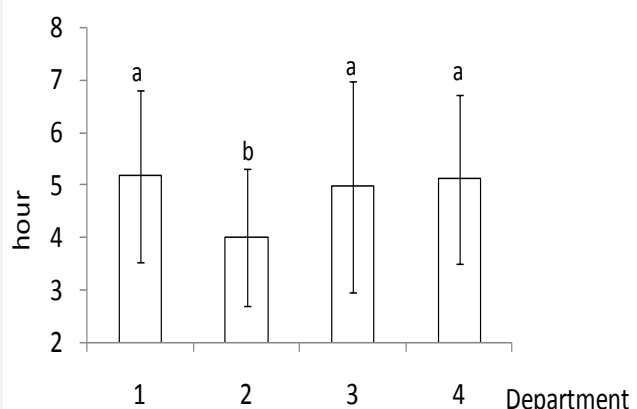

图 1A.男女大学生使用手机时间比较; 1B 不同系部学生使用手机时间比较 (1.护理系, 2. 口腔系, 3.药学系, 4. 食品营养系)；不同字母标注表示差异显著（ $p<0.05$, Fisher 最小显著差数法）。

Figure 1. A Time spending on mobile phone by males and females students. 1B. Time spending on mobile phone by students from different departments. Columns marked with different letters are significantly different at $\mathrm{p}<0.05$ using Fisher's protected LSD test. Bars on the columns represent standard error. 1.Department of Nursing, 2. Department of Dental Education, 3.

Department of Pharmacy, 4. Department of Food Science and Nutrition

\section{1 .1 使用手机目的分析}

该模式内容为调查多选项，总的来说，学生使用手机用于社交活动的占比最高占 $90.0 \%$, 用 于娱乐的次之为占 $72.7 \%$, 用于学习的占 $70.3 \%$, 用于购物的占 $62.7 \%$ 。但不同类别学生群体 使用手机的意图存在不同, 在给定的几种手机使用目的选项调查部分, 学生普通认可手机的 社会交流功能, 女生对列出的手机用途认可都比男生高。在分系部调查学生认可的手机功能 时, 护理系学生能对手机的学习功能最认可, 护理系学生使用手机购物的认可度也高于其它 三个系部（Table 1)。 
表1. 大学生使用手机用途调查结果（数据为选项人数占总调查人数的百分比; \%)。

Table 1 The purposes of students use mobile phone. The values were percentage of the choice number to the group $(\%)$.

\begin{tabular}{|c|c|c|c|c|c|}
\hline $\begin{array}{l}\text { Group } \\
\text { 分组 }\end{array}$ & $\begin{array}{l}\text { Social activity } \\
\text { 社交活动 }\end{array}$ & $\begin{array}{c}\text { Entertainment } \\
\text { • 娱乐 }\end{array}$ & $\begin{array}{l}\text { Shopping } \\
\text { 购物 }\end{array}$ & $\begin{array}{l}\text { Study } \\
\text { 学习 }\end{array}$ & $\begin{array}{l}\text { Other } \\
\text { 其它 }\end{array}$ \\
\hline $\begin{array}{l}\text { Male } \\
\text { 男生 }\end{array}$ & 83.33 & 58.33 & 50.00 & 52.78 & 11.11 \\
\hline $\begin{array}{l}\text { Female } \\
\text { 女生 }\end{array}$ & 91.33 & 75.72 & 65.32 & 73.99 & 12.14 \\
\hline $\begin{array}{l}\text { Nursing } \\
\text { 护理系 }\end{array}$ & 89.19 & 83.78 & 78.38 & 94.59 & 16.22 \\
\hline $\begin{array}{l}\text { Dental } \\
\text { 口腔系 }\end{array}$ & 85.54 & 67.47 & 59.04 & 66.27 & 6.02 \\
\hline $\begin{array}{l}\text { Pharmacy } \\
\text { 药学系 }\end{array}$ & 94.74 & 78.95 & 60.53 & 63.16 & 7.89 \\
\hline $\begin{array}{l}\text { Food and Nutrition } \\
\text { 食品营养系 }\end{array}$ & 94.12 & 68.63 & 58.82 & 64.71 & 21.57 \\
\hline
\end{tabular}

\section{1.2 手机引进身体不适症状情况分析}

大学生能够清楚的认识到过度使用手机能够对身体造成伤害, 调查身体相关部位出现不适率

（与总调查人数相比）分别是眼睛 $(70.33 \%) 、$ 肩 $(22.00 \%)$ 、背 $(11.18 \%)$ 、颈 $(32.54 \%)$ 、 头昏 (33. 49\%) 、指腕 (8.13\%) ，其它占 6.70\%等。同一调查对象不适症状累加计算结果如 表 2 。数据表明女生在眼、肩、颈部出现不适的情况比男生严重, 但男生出现的头昏涨和指 腕痛情况比女生严重, 这可能因为男生参与激烈刺激的手机游戏较多, 而女生用手机重点在 社交活动, 影视欣赏、购物等活动有关。从系部分类比较来看, 医学相关专业的学生如护理 系、口腔系学生较为注意用眼健康, 护理系以女生为主的群体, 用手机在学习上的时间较多, 头肩颈出现不适较少, 但学生课程多, 学习压力大, 手指力量较弱, 表现在问卷上头昏, 手 指酸痛等症状, 食品专业学生除头昏标准较低, 其它症状均最高, 表明手机对健康的负影响 更大 (Table 2)。

表2. 大学生使用手机过程产生不适症状分项调查（数据为选项人数占总调查人数的百分比; \%)。

Table 2 The symptoms caused by misusage of mobile phone, the value were percentage of the choice number to the group $(\%)$.

\begin{tabular}{|c|c|c|c|c|c|c|c|}
\hline $\begin{array}{l}\text { Group } \\
\text { 分组 }\end{array}$ & $\begin{array}{l}\text { Ophthalmodynia } \\
\text { 眼痛 }\end{array}$ & $\begin{array}{l}\text { Omodynia } \\
\text { 肩痛 }\end{array}$ & $\begin{array}{l}\text { Backache } \\
\text { 背痛 }\end{array}$ & $\begin{array}{l}\text { Cervicodynia } \\
\text { 颈痛 }\end{array}$ & $\begin{array}{l}\text { Vertigo } \\
\text { 头昏脑涨 }\end{array}$ & $\begin{array}{l}\text { Fingerache } \\
\text { 指腕痛 }\end{array}$ & $\begin{array}{l}\text { Other } \\
\text { 其它 }\end{array}$ \\
\hline $\begin{array}{l}\text { Male } \\
\text { 男生 }\end{array}$ & 58.33 & 19.44 & 11.11 & 27.78 & 41.67 & 13.89 & 8.33 \\
\hline $\begin{array}{l}\text { Female } \\
\text { 女生 }\end{array}$ & 72.83 & 22.54 & 11.56 & 33.53 & 31.79 & 6.94 & 6.36 \\
\hline $\begin{array}{l}\text { Nursing } \\
\text { 护理系 }\end{array}$ & 60.53 & 18.42 & 10.53 & 26.32 & 42.11 & 13.16 & 7.89 \\
\hline $\begin{array}{l}\text { Dental } \\
\text { 口腔系 }\end{array}$ & 67.07 & 18.29 & 9.76 & 34.15 & 28.05 & 6.10 & 6.10 \\
\hline $\begin{array}{l}\text { Pharmacy } \\
\text { 药学系 } \\
\text { Food and }\end{array}$ & 78.95 & 23.68 & 10.53 & 28.95 & 42.11 & 5.26 & 10.53 \\
\hline $\begin{array}{l}\text { Nutrition } \\
\text { 食品营养系 }\end{array}$ & 76.47 & 29.41 & 15.69 & 37.25 & 29.41 & 9.80 & 3.92 \\
\hline
\end{tabular}

以出现不适症状累加后, 进行不同组间的方差分析, 如图 3 所示, 在综合手机症状比较时, 男 女学生症状比较差异不显著 (Fig. 2), 不同系部综合症状比较存在显著差异, 口腔系学生症 状情况显著底于食品营养系。 

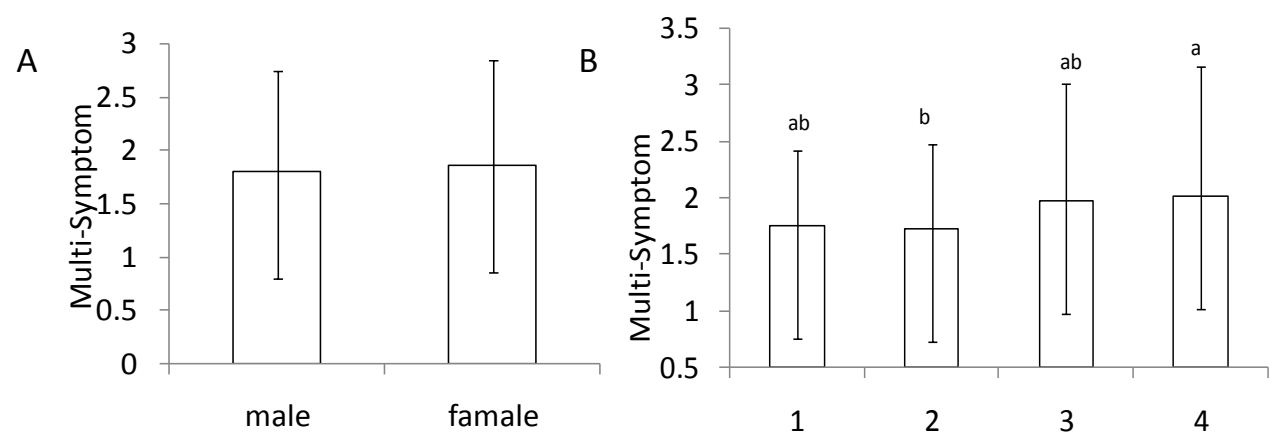

图2A. 男女大学生使用手机过度产生症状综合比较; $2 \mathrm{~B}$ 不同系部学生大学生使用手机过度产生症状综合比 较 (1. 护理系, 2. 口腔系, 3. 药学系, 4. 食品营养系) ; 不同字母标注表示差异显著 ( $p<0.05$, Fisher 最小 显著差数法)。

Figure 2A. Multi-Symptom present in males and females students. 2B. Multi-Symptom present in students from different departments. Columns marked with different letters are significantly different at $\mathrm{p}<0.05$ using Fisher's protected LSD test. Bars on the columns represent standard error. 1.Department of Nursing, 2. Department of Dental Education, 3. Department of Pharmacy, 4. Department of Food Science and Nutrition.

\section{2. 学生的体育运动情况}

问卷调查了学生每周运动时间，如表 3 所示，数据显示男生比女生运动时间较多，但未达显 著水平。不同系部的学生在体育运动投入时间上差异显著, 护理系和食品营养系的学生体育 运动时间显著少于口腔系。

Table 3 Hours of exercise spend by students of different groups weekly. Values marked with different letters are significantly different at $p<0.05$ using Fisher's protected LSD test.

\begin{tabular}{|c|c|}
\hline $\begin{array}{l}\text { Group } \\
\text { 分组 }\end{array}$ & Time(Hour) \\
\hline $\begin{array}{l}\text { Male } \\
\text { 男生 }\end{array}$ & $2.1875^{\mathrm{ab}}$ \\
\hline $\begin{array}{l}\text { Female } \\
\text { 女生 }\end{array}$ & $1.790462^{\mathrm{ab}}$ \\
\hline $\begin{array}{l}\text { Nursing } \\
\text { 护理系 }\end{array}$ & $1.493243^{\mathrm{b}}$ \\
\hline $\begin{array}{l}\text { Dental } \\
\text { 口腔系 }\end{array}$ & $2.201807^{\mathrm{a}}$ \\
\hline $\begin{array}{l}\text { Pharmacy } \\
\text { 药学系 }\end{array}$ & $1.967105^{\mathrm{ab}}$ \\
\hline $\begin{array}{l}\text { Food and Nutrition } \\
\text { 食品营养系 }\end{array}$ & $1.485294^{\mathrm{b}}$ \\
\hline
\end{tabular}

3. 3. 学生体育运动与手机依赖的相关性分析

经对学生手机使用时间, 体育运动时间及手机相关综合症状相关性进行了分析, 结果如下表 所示, 学生手机使用时间与手机产生综合症状相关性极显著（ $p<0.01 ） 。$ 本次调查结果中体 育运动多为自发开展（只是口腔系的学生由系部发动了远离手机活动），学生的体育运动时 间与学生的手机使用时间、手机所致综合症状负相关极弱（Table 3）。 
表 3. 手机使用时间、体育运动时间和手机使用不适综合症状相关性分析 ( $* *$ 标注表示极显著, $p=0.01 ; *$ 标注表示显著, $p$ $=0.05$ )。

Table 4. Correlation among the mobiletime and exercise time spent by students and their multi-symptom caused by mobile usage. **. Showed the significant difference at level of $\mathrm{p}=0.01$

((2-tailed), *. Showed the significant difference at level of $\mathrm{p}=0.05$ ((2-tailed).

\begin{tabular}{|c|c|c|c|c|c|c|}
\hline & & & & Exercise time & Mobiletime & Multi-Symptom \\
\hline \multirow{7}{*}{ Exercise time } & \multicolumn{3}{|c|}{ Pearson 相关性 } & 1 & -.058 & -.086 \\
\hline & \multicolumn{3}{|c|}{ 显著性（双侧） } & & .403 & .216 \\
\hline & \multicolumn{3}{|l|}{$\mathrm{N}$} & 209 & 209 & 209 \\
\hline & \multirow{4}{*}{ Bootstrap $^{c}$} & \multicolumn{2}{|l|}{ 偏差 } & 0 & -.001 & -.001 \\
\hline & & \multicolumn{2}{|l|}{ 标准 误差 } & 0 & .063 & .059 \\
\hline & & \multirow{2}{*}{ 95\% 置信区间 } & 下限 & 1 & -.183 & -.193 \\
\hline & & & 上限 & 1 & .065 & .039 \\
\hline \multirow{7}{*}{ Mobiletime } & \multicolumn{3}{|c|}{ Pearson 相关性 } & -.058 & 1 & $.293^{* *}$ \\
\hline & \multicolumn{3}{|c|}{ 显著性 ( 双侧 ) } & .403 & & .000 \\
\hline & \multicolumn{3}{|l|}{\begin{tabular}{|l|}
$\mathrm{N}$ \\
\end{tabular}} & 209 & 209 & 209 \\
\hline & \multirow{4}{*}{ Bootstrap $^{c}$} & \multicolumn{2}{|l|}{ 偏差 } & -.001 & 0 & .004 \\
\hline & & \multicolumn{2}{|l|}{ 标准 误差 } & .063 & 0 & .084 \\
\hline & & & 下限 & -.183 & 1 & .123 \\
\hline & & & 上限 & .065 & 1 & .455 \\
\hline \multirow{7}{*}{$\begin{array}{l}\text { Multi-Sympto } \\
\text { m }\end{array}$} & \multicolumn{3}{|c|}{ Pearson 相关性 } & -.086 & $.293^{* *}$ & 1 \\
\hline & \multicolumn{3}{|c|}{ 显著性 ( 双侧 ) } & .216 & .000 & \\
\hline & \multicolumn{3}{|l|}{$\mathrm{N}$} & 209 & 209 & 209 \\
\hline & \multirow{4}{*}{ Bootstrap $^{c}$} & \multicolumn{2}{|l|}{ 偏差 } & -.001 & .004 & 0 \\
\hline & & \multicolumn{2}{|l|}{ 标准 误差 } & .059 & .084 & 0 \\
\hline & & \multirow{2}{*}{ 95\% 置信区间 } & 下限 & -.193 & .123 & 1 \\
\hline & & & 上限 & .039 & .455 & 1 \\
\hline
\end{tabular}

\section{4. 讨论与结论}

4. 1. 运动时间和强度与手机使用时间的关系

通过调查发现， 209 人中 169 人每次锻炼时间在 1 小时以下，每周 1-3 次或 3 次以上，那么 每天锻炼的时间不足 0.5 小时，对比 66.5 的学生每天使用手机在 5 小时以上，学生运动时间 和手机使用时间严重的不对等, 学生锻炼身体的运动时间非常少。另外调查发现, 在每天使 用手机在 8 小时的 29 名学生中, 有 13 人选择了偶尔参加锻炼, 且每次锻炼时间均在 1 小时 以下; 每天使用手机时间在 5 小时的 109 名学生中，有 29 人选择了偶尔参加锻炼， 24 人选 择了每周 1-3 次，且每次锻炼时间均在 1 小时以下; 而在每周锻炼次数在 3 次以上且每次不 低于 2 小时的 21 人中，全部上网时间低于 5 小时。由此可见，使用手机的时间和参加体育锻 炼的时间是呈反向表现的, 手机使用时间越长, 参加锻炼的时长就越短。但因为学生的体育 运动多自发开展, 形式和时间差异较大。我们试图寻找体育运动时间和手机使用时间的关系, 但学生在校学习时时间的利用有学习、社会实验、社团活动、休息等及有规律的时间安排外, 学生其余时间安排随意性很大, 对于体育运动的实践明确缺少规律, 因此要加强引导 [4-5]。 至于运动内容, 教育到眼和颈部症状高发, 头昏现象也较严重, 男生指腕等症状高发, 体育 的引导应多开展户外，特别是能舒展上肢的体育项目。

4.2. 积极对学生加强引导 
大学生因其活泼敏感, 易受到外界影响, 特别是同学之间, 不同系部之间的学生用手机目的 的差异, 班极系部开展的活动如口腔系的 “远离手机一别把青春交给手机” 活动, 在上课时 把手机离身放置的要求, 对本次调查中手机时间一项中产生了显著影响。口腔系学生从用手 机时间, 综合症状及运动时间上均强于食品营养系和药学系。同是医学相关专业的护理系学 生认同手机使用的学习目的, 在手机时间上较长, 体育运动时间较少, 但可能专业特点, 学 生的保健意思使学生注意了使用手机的姿势保持正确, 综合症状标准较好。食品营养系及药 学系在手机的正确使用上更要加强引导，特别是自我控制时间，正确坐立行的姿势。

\section{参考文献}

[1] 王欢, 黄海, 吴和鸣 WANG Huan, HUANG Hai, WU He-ming. 大学生人格特征与手机依赖的 关系：社交焦虑的中介作用 Relationship between Personality and Mobile Phone Addiction: A Mediating Role of Social Anxiety. [J] 中国临床心理学杂志 Chinese Journal of Clinical Psychology ,2014, 22 (3) :447-450

[2] 李丽，牛志民，梅松丽，宋玉婷 LI Li，NIU Zhi-min，MEI Song-1i，SONG Yu-ting. 医 学生智能手机成瘾, 冲动性与心理健康相关分析 Relationship between smartphone addiction, impulsivity and mental health among medical students. [J]现代预防 医学 Modern Preventive Medicine ,2016, 43 (3):482-485

[3] 冯桂梅, 王晓英 FENG Gui-Mei; WANG Xiao-Ying. 90 后大学生手机依赖症心理分析及对 策研究After the 90 College Students Mobile Phone Addiction Psychological Analysis and Prevention Countermeasures. [J] 中国卫生产业 CHI NA HEALTH INDUSTRY, 2015, 139-141

[4] 刘亚丽，黎丹丹 Liu Yali;Li Dandan. 大学生手机依赖者的心理渴求感 Psychological Craving Sense of Mobile Phone Dependency Among College Students. [J]心理研究 Psychological Research, 2016, 9 (1):79-84

[5] Lingjie 薛王高韩任 XWYGNHYR 大学生手机依赖的形成机制及应对策略研究 Study on formation machanism of mobile phone dependence of college students and countermeasures. [J]浙江理工大学学报 (社会科学版 Journal of Zhejiang Sci-Tech University (Social Sciences) , 2016, 36 (1):90-95

作者简介: 尹军杰 (1978 年一), 男, 河南临颖, 高校讲师, 主要研究方向--体育教育, E-mail: 1079345161@qq.com。 